\title{
周环上芳基取代的叶绿素类二氢卟吩衍生物的合成
}

\author{
于沙沙 ${ }^{a}$ 徐希森 ${ }^{b}$ 刘 洋 $b$ 李家柱 ${ }^{b}$ 金英学 $*, a$ \\ 祁彩霞 $^{c}$ 王进军 ${ }^{*}, b, c$ \\ $\left({ }^{a}\right.$ 哈尔滨师范大学化学化工学院 哈尔滨 150025) \\ ( $b$ 烟台大学化学化工学院 烟台 264005) \\ ( ${ }^{c}$ 山东省黄金工程技术研究中心(工业应用) 烟台 264005)
}

\begin{abstract}
摘要 以叶绿素降解产物脱镁叶绿酸-a 甲酯为起始原料, 利用其二氢卟吩大环上的活性反应区域, 沿着 $\mathrm{N}^{21}-\mathrm{N}^{23}$ 轴向进 行官能团修饰, 对五元外接 E-环也实施了结构改造, 在周环上分别引进了能与大环色基形成不同共轭程度的芳香性取 代基团, 完成了 12 个未见报道的叶绿素类二氢卟吩衍生物的合成, 其化学结构均经 UV, IR, ${ }^{1} \mathrm{H}$ NMR 和质谱及元素分 析予以证实, 同时也讨论了芳基的引进对二氢卟吩的理化性质所产生的各种影响.
\end{abstract}

关键词＼cjkstart叶绿素-a; 二氢卟吩; 化学修饰; 芳基取代; 合成

\section{Synthesis of Chlorophyllous Chlorins Derivatives Substituted by Aromatic Groups on Their Periphery}

\author{
Yu, Shasha ${ }^{a}$ \\ $\mathrm{Xu}, \mathrm{Xisen}^{b}$ \\ Liu, Yang ${ }^{b}$ \\ Li, Jiazhu ${ }^{b}$ \\ Jin, Yingxue ${ }^{*, a}$ \\ Qi, Caixia \\ Wang, Jinjun ${ }^{*, a, b}$ \\ $\left({ }^{a}\right.$ College of Chemistry \& Engineering, Harbin Normal University, Harbin 150025) \\ ( ${ }^{b}$ College of Chemistry and Chemical Engineering, Yantai University, Yantai 264005) \\ ( ${ }^{c}$ Shandong Applied Research Centre of Gold Nanotechnology (Au-SDARC), Yantai 264005)
}

\begin{abstract}
Pyropheophorbide-a methyl ester, as a degraded product from chlorophyll-a, was used as a starting material, and the modifications for functional group along $\mathrm{N}^{21}-\mathrm{N}^{23}$-axis and reconstructions for five-membered exocyclic E-ring were completed to introduce different aromatic groups which could conjugated with macrocyclic chromophore at various degrees on the periphery. The syntheses of 12 unreported chlorins related to chlorophyll were accomplished and their chemical structures were characterized by elemental analysis, UV, IR, MS and ${ }^{1} \mathrm{H}$ NMR spectra. The different effects on the physical and chemical properties of chlorophyllous chlorins by introduction of aromatic groups were discussed.
\end{abstract}

Keywords chlorophyll-a; chlorin; chemical modification; substitution of aromatic group; synthesis

叶绿素(Chls)和细菌叶绿素(BChls)的降解产物作为 光动力治疗 (Photodynamic therapy, 简称 PDT)的理想前 体一直受到人们的关注, 其优良的光物理和光生物性质 完全满足用作 PDT 抗癌药物所需要的基本要求, 特别 是叶绿素类二氢卟吩所具有的最大波长吸收谱带 $(\mathrm{Qy})$, 标识着四吡咯大环分子在生物组织的穿透能力以及对 单线态氧的激发程度 ${ }^{[1 \sim 3]}$. 利用 $\mathrm{N}^{21}-\mathrm{N}^{23}$ 轴向两端和 20-meso-位的结构修饰, 构建不同的化学结构和取代基
团, 在 PDT 药物合成研究中日益受到人们的热切关

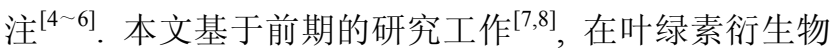
周环的不同位置上引进可能带来特殊理化性质的芳基 结构, 旨在进一步改善大环分子光谱性质, 合成更多具 有应用前景的叶绿素类二氢卟吩衍生物.

以焦脱镁叶绿酸-a 甲酯 $(\mathbf{1}$, 简称 MPPa) 为起始原料, 选择由四氧化锇和高碘酸钠所组成的混合氧化剂对其 3-位乙烯基进行氧化, 生成的焦脱镁叶绿酸-d 甲酯再经

*E-mail: wjj1955@163.com

Received July 31, 2013; revised October 11, 2013; published online October 25, 2013.

Project supported by the National Natural Science Foundations of China (No. 21272048) and the Project of Shandong Applied Research Centre of Gold Nanotechnology (2011).

国家自然科学基金(No. 21272048)和山东省黄金工程技术研究中心(2011 年度)资助项目. 
$N$-氯代琥珀酰亚胺(NCS)氯代, 选择性地给出 20 -氯代 二氢卟吩醛 $(2,46 \%)$, 再与氯化苄基三苯基鏻进行 Wittg 反应, 以约 $3: 1$ 的比例给出 $3 b$-位 $E / Z$-苯基取代的焦脱 镁叶绿酸 3a (8\%) 和 $3 \mathbf{b}(26 \%)$, 同时还分离出 $4 \%$ 的 $132-$ 位氧化的 Wittg 反应产物(3c). 在 $\mathrm{LiOH}$ 促进的空气氧化 反应中, MPPa (1)的外接 E-环被顺利地氧化成环戊二酮 结构, 再于 NBS 和甲醇钠共存条件下, 对硝基苯甲醛肜 转化成氧化对硝基苯甲腈, 进而与所得二氢卟吩二酮 (4)的 3-位乙烯基发生 1,3-偶极环加成反应，分别以 21\% 和 10\%的产率分离出 3-位异噁唑啉基取代的二氢卟吩 二酮 5 和红紫素-18 衍生物 6 . 化合物 1 与邻氨基苯甲腈 的 Friedlaender 反应, 仅给出未关环的 $13^{2}$-位氧化和 $13^{1}$ 位亚胺化的二氢卟吩衍生物 7 (47\%) (Scheme 1).

选择 $3 b$ - 苯基焦脱镁叶绿酸-a 甲酯 (8) 为反应物 ${ }^{[9]}$, 经碱催化的空气氧化得到二氢卟吩二酮 (9), 继续与邻 苯二胺反应后, 主要环合成喹诺啉并焦脱镁叶绿酸(10) (17\%)和苯并咪唑并红紫素-18 衍生物 11 (28\%); 12-甲酰 基焦脱镁叶绿酸-a 甲酯(12)的 C-子环上连有两个羰基, 考虑到与邻苯二胺有可能缩合成环, 用 4-苯甲酰基邻苯 二胺与其进行环合, 同样得到甲酰基与氨基缩合的 $12-N$-苯亚氨基取代的焦脱镁叶绿酸-a 甲酯 13 (38\%). 红紫素-18 (14)与对氨基芐胺的酰胺化反应高产率地给
出红紫素-18 亚酰胺 $(\mathbf{1 5}, 71 \%)$; 与水合联胺发生肼解后 再与对苯二甲醛进行缩合, 得到 $42 \%$ 的分子端位连有甲 酰基的红紫素-18 亚酰胺(16a); 与盐酸着弪胺的氨解产物 进一步与苯甲酰氯反应, 分离出 $56 \%$ 的 $N$-苯甲酰氧基 红紫素-18 酰亚胺(16b) (Scheme 2).

\section{1 结果与讨论}

\section{1 芳基的引进与二氢卟吩的立体化学结构}

二氢卟吩醛 2 与氯化芐基三苯基鏻的 Wittig 反应给 出一对 $E / Z$ 异构体 $\mathbf{3 a}$ 和 $\mathbf{3 b}$ 和另一 $\mathrm{E}-$ 环氧化产物 $\mathbf{3 c}$, 根 据它们烯键上连在 $3 a-$ 和 $3 b-$ 位氢质子间的偶合常数, 可 以确定反应产物的顺反异构. 在 Wittig 反应中, 虽然 3a 和 3b 的比例难于控制, 但其 3a-位的立体结构能够发生 转换. 如果将混合物于室温下放置 $45 \mathrm{~d}$ 以上, 顺式异构 体 $3 \mathrm{a}$ 基本可以全部转化成反式异构体 $\mathbf{3 b}$. 化合物 $\mathbf{3 c}$ 是 E-环空气氧化产物, 而有氧和碱性是空气氧化的必要反 应条件 ${ }^{[10 a]}$, 如果 Wittig 反应的时间越长, $\mathbf{3 c}$ 的产率则越 高. 与此相同, 化合物 $\mathbf{6}$ 是 1,3-偶极环加成反应中的 E环空气氧化反应产物. 所以, 良好的空气隔绝和缩短碱 性环境接触可以在一定程度上降低 $3 \mathrm{c}$ 和 6 的产率. 从 $3 \mathbf{a}$ 和 $\mathbf{3 b}$ 的氢谱中可以发现，除了偶合常数的差异以外，

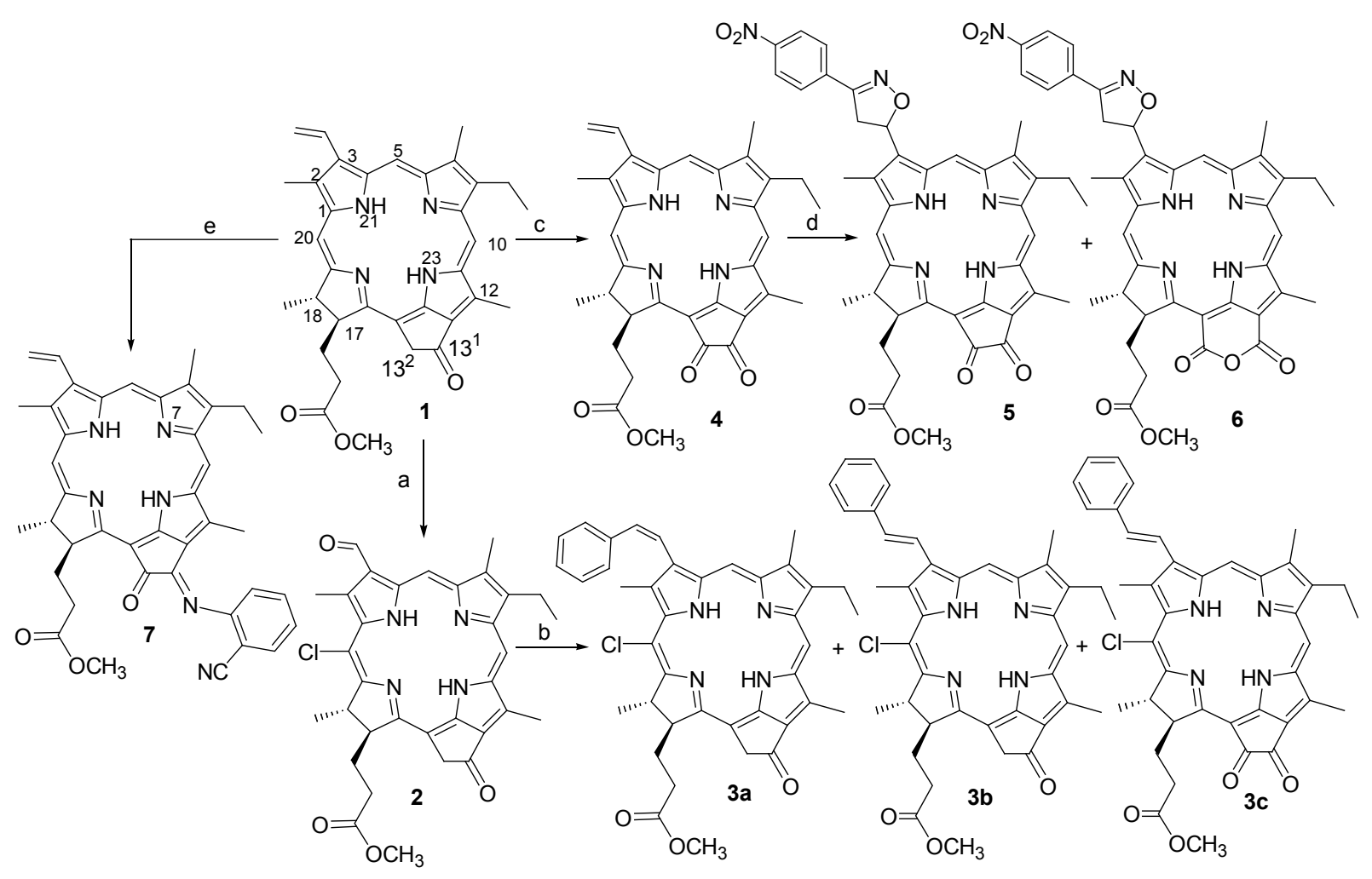

Reagents and conditions: (a) $\mathrm{OsO}_{4} / \mathrm{NalO}_{4} / \mathrm{NCS} / \mathrm{CH}_{2} \mathrm{Cl}_{2}$; (b) $\mathrm{Ph}_{3} \mathrm{PCH}_{2} \mathrm{Ph} / \mathrm{CH}_{2} \mathrm{Cl}_{2} / \mathrm{NaOH}$; (c) $\mathrm{LiOH} / \mathrm{O}_{2} / \mathrm{THF} / \mathrm{MeOH}$; (d) $p-\mathrm{NO}_{2} \mathrm{C}_{6} \mathrm{H}_{4} \mathrm{C}=\mathrm{NOH} / \mathrm{NBS} / \mathrm{NaOCH}_{3} ;$ (e) o- $\mathrm{NH}_{2} \mathrm{C}_{6} \mathrm{H}_{4} \mathrm{CN} / \mathrm{Pyr} / \mathrm{TFA}$

Scheme 1 


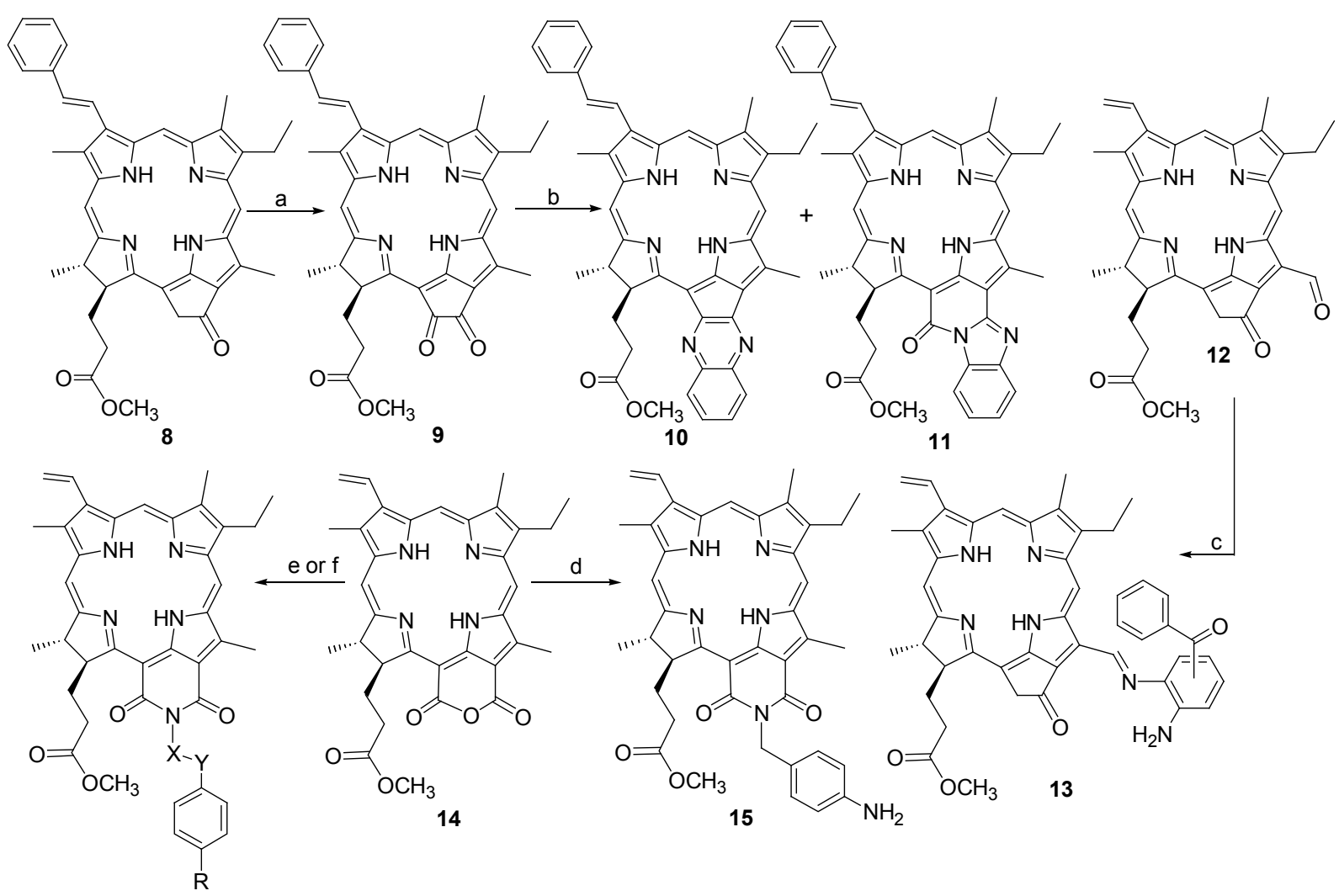

16a: $X-Y=N=C H, R=C H O$;

16b: $X-Y=O C=O, R=H$

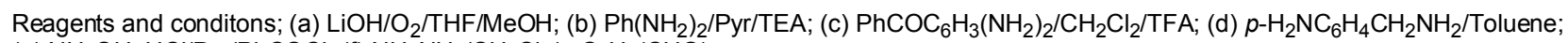
(e) $\mathrm{NH}_{2} \mathrm{OH} \cdot \mathrm{HCl} / \mathrm{Pyr} / \mathrm{PhCOCl}$; (f) $\mathrm{NH}_{2} \mathrm{NH}_{2} / \mathrm{CH}_{2} \mathrm{Cl}_{2} / p-\mathrm{C}_{6} \mathrm{H}_{4}(\mathrm{CHO})_{2}$

\section{Scheme 2}

$3 \mathbf{b}$ 的 3a-位质子出现在相对低场 $(\delta=8.29)$, 而 $Z$-式异构 体的相同质子则高场移动至 $\delta 7.66$ 处. 由于 $E$-式结构的 $3 \mathrm{~b}$-位上苯基与色基相距较远, 与其可以形成的良好共 平面, 3a-位质子能够强烈地承受到来自二氢卟吩大环的 去屏蔽作用; 而 3a 的苯基靠近四吡咯大环，相互间的排 斥作用促使二者沿着 $\mathrm{C}(3)-\mathrm{C}(3 \mathrm{a})$ 轴发生旋转, 其偏移 的结果使得 $3 \mathrm{a}$-位氢原子与大环形成一定的二面角(图 1). 因此, 氮杂轮烯的环电流对其形成的去屏蔽作用相 对较弱; 远离大环的 3b-质子主要受到苯基的去屏蔽效 应, 所以 $3 \mathrm{a}$ 和 $3 \mathrm{~b}$ 的 $3 \mathrm{~b}$-位上质子吸收均出现在同一位 置 $(\delta=7.49)$.

化合物 7 中以亚胺键价形式连接的苯基在外接环上 也形成了 $E / Z$ 结构, 其氢谱中的许多质子均给出成对的 吸收峰值. 从 $E / Z$ 异构体的质子积分估算, 二者的比例 大约为 $5: 2$; 与 $E$-式结构相比, $Z$-式异构体中的苯基更 加靠近 132-位, 原子间的相互排斥使得 $E$-环羰基向 17位偏移, 其碳氧双键对 17-位质子实施更为有效的去屏 蔽作用, 从而促成其化学位移发生低场移动 $(\delta=5.24)$, 而 $E$-式中的相应吸收峰则出现在 $\delta=5.14$ 处.

酰亚胺结构中的氮上取代基团围绕与其所形成的
化学键旋转可能受到一定程度的限制. 化合物 15 中与 氮连接的碳原子是 $\mathrm{sp}^{3}$ 杂化，除了 $\mathrm{C}-\mathrm{N}$ 键以外，另外三 个 $\mathrm{sp}^{3}$ 键分别与氢原子和苯基相连, 其翻转伞状结构在 旋转时与两个羰基始终保持平行状态, 因此不存在空间 位阻问题. 红紫素亚酰胺-18 16a 中与氮相连的是 $\mathrm{sp}^{2}$ 杂 化的氮原子，与醛基碳原子形成了 $E$-式 $\mathrm{C}=\mathrm{N}$ 双键，另 一非键的 $\mathrm{sp}^{2}$ 轨道上承载着一对孤对电子, 在旋转时氮 上取代部分也可形成相似的翻转伞状结构. 尽管亚胺碳 上所连氢原子的指向靠近酰亚胺羰基, 但因其原子半径 太小而不能阻碍绕 $\mathrm{N}-\mathrm{N}$ 键的正常转动. 如果 $\mathrm{C}=\mathrm{N}$ 双 键上的芳基时以 $Z$-式结构存在，显然不能顺利地完成 $\mathrm{N}-\mathrm{N}$ 键的绕轴旋转, 所以, 没有形成阻旋异构也间接 地证明了其亚胺键具有 $E$-式结构. 化合物 $\mathbf{1 6 b}$ 中的亚酰 胺氮的上氢被苯甲酰氧基所取代, 虽然从空间取向上看 与 $16 \mathrm{a}$ 大致相同, 但当苯甲酰羰基绕轴旋转到与亚酰胺 共平面时, 其羰基氧原子的两个非键 $\mathrm{sp}^{2}$ 轨道与酰亚胺 羰基碳上的孤对电子发生重叠, 因此形成一对难于分离 的阻旋异构体，核磁共振氢谱中呈现出的成对峰值印证 了上述的讨论. 

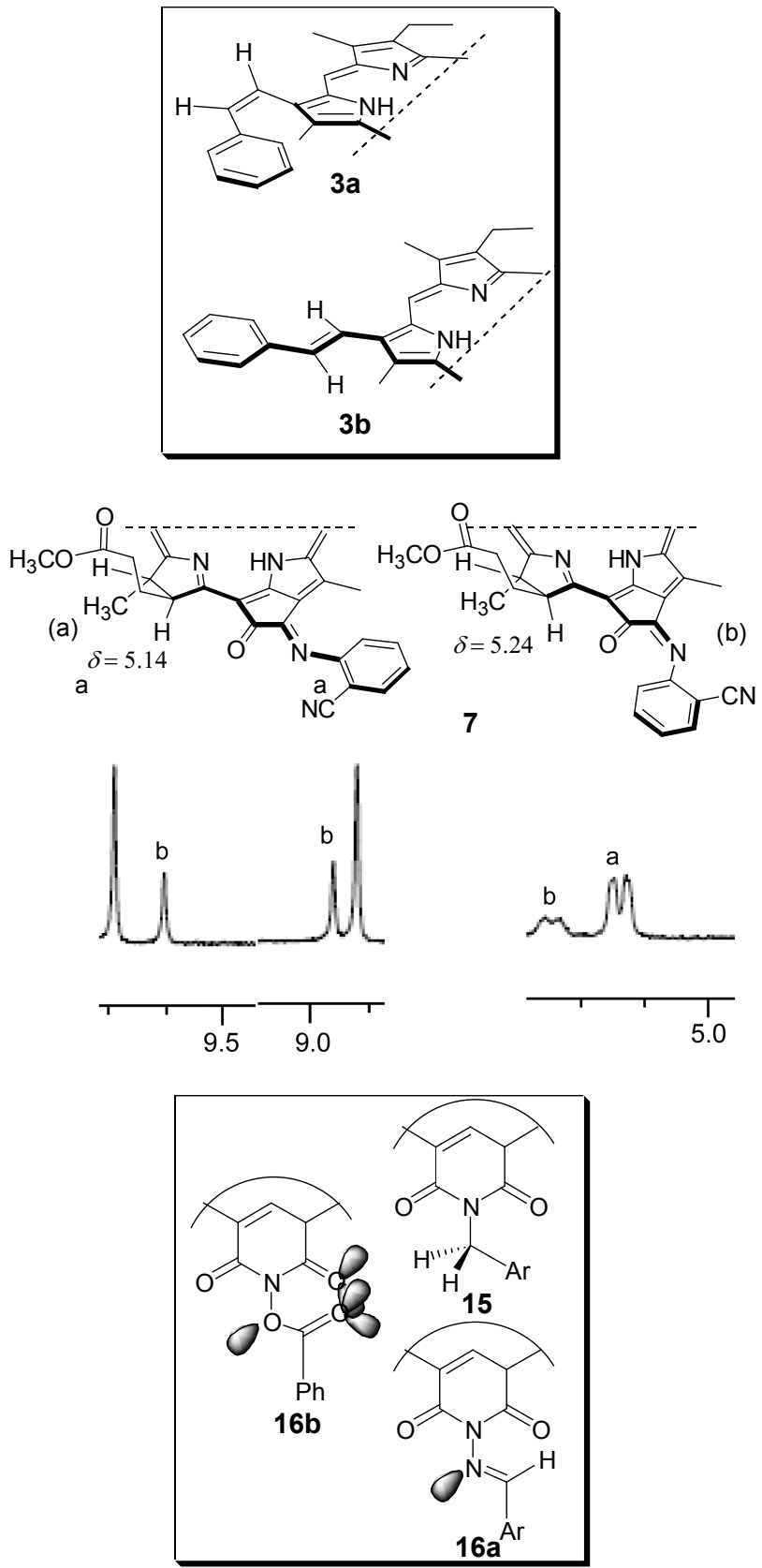

图 1 芳基的空间取向与其化学位移

Figure 1 The spatial configurations of aromatic groups and their chemical shifts

\section{3 芳基的引进与二氢卟吩的紫外-可见光谱}

紫外-可见光谱是叶绿素类二氢卟吩在光医学等诸 多应用方面所要考量的重要参数, 而不同位置上的芳基 引进可以明显地改善大环分子的光物理性质, 特别是沿 着 $\mathrm{N}^{21}-\mathrm{N}^{23}$ 轴向的化学结构修饰对最大可见光的吸收具 有强烈地影响 ${ }^{[10 b]}$. 化合物 3 中 C(3)-甲酰基的 Wittg 反 应在 3b-位上引进苯基, 扩展了四吡咯分子中芳香性氮 杂轮烯的共轭体系. 与 20-氯代焦脱镁叶绿酸-a 甲酯相 比 $^{[11]}, \mathbf{3 a}$ 和 $\mathbf{3 b}$ 的最大可见光吸收分别红移了 3 和 $8 \mathrm{~nm}$, 后者之所以发生更大幅度的红移, 其原因可以归结为处
于 $E$-式结构的苯基与大环形成较好的共平面，通过 3位碳碳双键有效地参了与二氢卟吩色基的共轭体系; 而 $Z$-式异构体的 3b-位上苯基与大环色基的排斥作用促使 其偏离共平面，因而苯基不能充分地与大环共轭，其结 果缩短了最大可见光的延长距离. 与 $\mathrm{MPPa}(\mathbf{1})$ 的最大可 见光的吸收波长 $(668 \mathrm{~nm})$, 苯基取代的叶绿素衍生物的 Qy 吸收均向长波方向发生不同程度的推移. 认真观察 变化的幅度可以看出, 所形成的吸收差异 $(\Delta \mathrm{Qy})$ 则不尽 相同. 其中, 在 $3 \mathrm{~b}$-位和外接环上双向引入芳基的二氢 卟吩 10 和 11 的红移距离相对较长 (46 和 $62 \mathrm{~nm}$ ), 而仅 $3 b$-位单向苯基取代的二氢卟吩 $3 \mathbf{b}, \mathbf{3 c}, \mathbf{8}$ 和 9 的 $\Delta \mathrm{Qy}$ 大 致范围为 $6 \sim 9 \mathrm{~nm}^{[11,12]}$ (图 2).

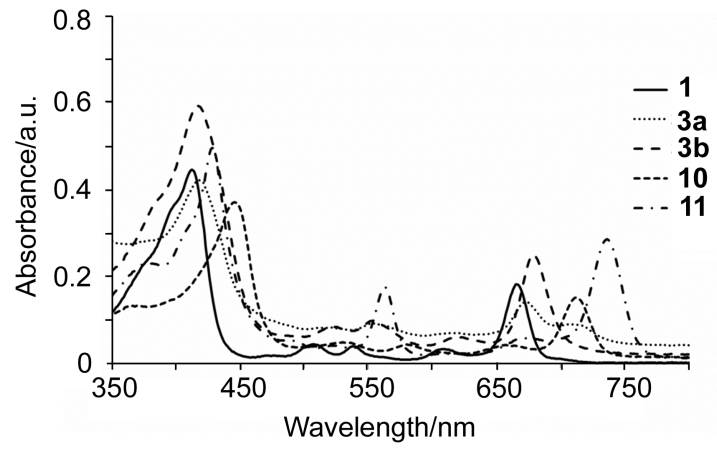

图 2 焦脱镁叶绿酸-a 甲酯 1 及其苯基取代二氢卟吩衍生物 3a, 3b, 10 和 11 的紫外-可见光谱

Figure 2 UV-Vis spectra of methyl pyropheophorbide-a 1 and its phenyl-substituted chlorin derivatives $\mathbf{3 a}, \mathbf{3 b}, \mathbf{1 0}$ and $\mathbf{1 1}$

$3 b$-位苯基的共轭效应对波长红移的贡献则相对较 小; 其 $\mathrm{C}(3)$-双键上的苯基是 $\mathrm{Qy}$ 延长的唯一策动力, 因 而红移的距离相对较短. 在 $\mathrm{N}^{21}-\mathrm{N}^{23}$ 轴向另一端的 C-环 上所连带的芳基, 同样可以高强度地提升最大可见光的 吸收波长, 例如, 在 $13^{2}$-位和 12 -位上连有苯亚胺结构的 二氢卟吩 7 和 13, 其最大可见光的吸收波长分别为 709 和 $693 \mathrm{~nm}$. 与二氢卟吩二酮 4 和起始原料 1 相比，相应 地向长波方向推移了 32 和 $28 \mathrm{~nm}^{[13]}$.

与直接与大环色基形成共轭的方式不同，虽然红紫 素-18 亚酰胺的氮上芳基均跨越多个单键与色基相连, 但是其最大可见光的吸收波长存在着明显的不同. 尽管 红紫素-18 亚酰胺外接环亚胺中的氮原子是以 $\mathrm{sp}^{2}$ 杂化 的方式存在 ${ }^{[14]}$, 但化合物 15 中氮上所连的对氨基苄基 与亚酰胺的碳氧双键相距较远, 且相连单键中还嵌有一 个 $\mathrm{sp}^{3}$ 杂化的碳原子，因而不能形成任何联系，其 Qy 吸 收值也仅为 $706 \mathrm{~nm}$ ，与氮上无取代同系物的相应数值 极为相似 $(705 \mathrm{~nm})^{[6 \mathrm{cc}]}$. 红紫素-18 亚酰胺 16a 的外接环芳 基通过亚胺的碳氮双键与亚酰胺环中的氮原子相连, 包 括苯基上的甲酰基，与色基相连的所有原子均为 $\mathrm{sp}^{2}$ 杂 化，因而可以沿着外接环与氮上芳基建立一个扩展的共 
轭体系, 将其最大可见光的吸收波长延至 $716 \mathrm{~nm}$. 相对 而言，尽管 $16 \mathrm{~b}$ 的氮上所连有的苯甲酰氧基的电子结构 满足与亚酰胺外接环形成共轭的条件, 但其阻旋异构现 象限制了与大环形成良好的共平面, 亚酰胺环中氮原子 所连的共轭结构难于与色基发生有效的电子离域, 最终 其最大可见光的吸收波长落到 $711 \mathrm{~nm}$.

\section{2 结论}

利用叶绿素类二氢卟吩芳香性大环上的活性反应 区域, 在周环 $\mathrm{N}^{21}-\mathrm{N}^{23}$ 轴向的不同位置上构建和引进了 芳香环结构, 可以有效地扩展大环分子的共轭体系, 并 促成其电子光谱产生规律性变化. 同时, 随着芳基的引 入在四吡咯环上形成新的反应区域, 并对原有反应位点 的活性也会产生一定程度的影响, 为进一步改变叶绿素 降解产物的化学结构, 完善其光物理、光生物等诸多性 质提供了有效的切入手段.

\section{3 实验部分}

\section{1 仪器与试剂}

元素分析用 Perkin-Elmer 2400 型元素分析仪测定; IR 用 Perkin-Elmer 1730 型红外分光光度仪测定 $(\mathrm{KBr}$ 压 片); UV-Vis 用 UV-160A 型紫外分光光度计测定; ${ }^{1} \mathrm{H}$ NMR 用 Brucker ARX-300 型核磁共振仪测定, 内标为 TMS; ESI-MS 用 Bruker Daltonics Esquire3000 plus 或 者 Varian 500-MS IT 质谱仪测定; 所用试剂均为分析纯 或化学纯. 焦脱镁叶绿酸-a 甲酯 1 按文献[15]制备; 132氧代焦脱镁叶绿酸-a 4、红紫素-18 甲酯 14 和 12-甲酰基 焦脱镁叶绿酸甲酯-a 12 按文献[12]; trans-3b-苯基焦脱 镁叶绿酸-a 甲酯 8 按文献[9]制备.

\subsection{0 -氯焦脱镁叶绿酸- $d$ 甲酯(2)的合成}

在 $25 \mathrm{~mL}$ 干燥的四氢呋喃中溶解 $139 \mathrm{mg} \mathrm{MPPa} 1$ (0.253 mmol), 再向溶液中加入 $0.5 \mathrm{~mL}$ 吡啶, 然后将体 系冷却至 $0{ }^{\circ} \mathrm{C}$, 剧烈摚拌的条件下, 迅速加入溶有 110 $\mathrm{mg}$ 四氧化锇的 $2 \mathrm{~mL}$ 四氢呋喃, 搅拌 $30 \mathrm{~min}$ 后, 升温至 室温, 在摚拌反应 $1 \mathrm{~h}$, 加入过量用亚硫酸氢钠饱和的 $50 \%$ 甲醇溶液并搅拌 $20 \mathrm{~min}$, 过滤除去沉淀, 滤液减压 浓缩至干并重新溶解于 $15 \mathrm{~mL}$ 甲醇中, 先后将加入溶解 于 $15 \mathrm{~mL}$ 水中 $500 \mathrm{mg} \mathrm{NaIO}_{4}$ 和 $1 \mathrm{~g}$ 硅胶, 反应体系的颜 色迅速由黑绿色变为铜色, $30 \mathrm{~min}$ 后反应完毕, 加入 $100 \mathrm{~mL}$ 水和 $80 \mathrm{~mL}$ 二氯甲烷, 静置过夜, 分出有机层, 水层用二氯甲烷萃取 $(15 \mathrm{~mL} \times 3)$, 合并有机层并用无水 硫酸钠干燥, 减压除尽溶剂, 将剩余物重新溶解于 150 $\mathrm{mL}$ 四氢呋喃中, 室温条件下慢慢滴加含有 $75 \mathrm{mg} \mathrm{NCS}$ 的 $10 \mathrm{~mL}$ 甲醇溶液, 于 $1.5 \mathrm{~h}$ 内滴加完毕, 再继续反应 4 h. 反应结束后, 先后加入 $30 \mathrm{~mL}$ 水和 $20 \mathrm{~mL}$ 二氯甲烷,
分出有机层，用无水硫酸钠干燥，浓缩，经硅胶柱层析 分离[洗脱剂: $V$ (石油醚) $: V($ 乙酸乙酯 $)=3: 1$ ], 得 68 $\mathrm{mg}$ 暗红色固体产物 2 (0.117 mmol), 产率 46\%. m.p. 236 239 ${ }^{\circ} \mathrm{C}$; UV-vis $\left(\mathrm{CH}_{2} \mathrm{Cl}_{2}\right) \lambda_{\max }\left[\varepsilon /\left(\mathrm{L} \cdot \mathrm{mol}^{-1} \cdot \mathrm{cm}^{-1}\right)\right]$ : $422\left(1.33 \times 10^{5}\right), 533\left(7.74 \times 10^{3}\right), 567\left(2.89 \times 10^{4}\right), 645$ $\left(1.71 \times 10^{4}\right), 703\left(7.65 \times 10^{4}\right) \mathrm{nm} ;{ }^{1} \mathrm{H}$ NMR $\left(\mathrm{CDCl}_{3}\right) \delta:$ -2.12 (br, 1H, NH), 0.65 (br, 1H, NH), 1.64 (t, $J=7.6 \mathrm{~Hz}$, $\left.3 \mathrm{H}, 8 \mathrm{~b}-\mathrm{CH}_{3}\right), 1.66$ (d, $\left.J=7.0 \mathrm{~Hz}, 3 \mathrm{H}, 18-\mathrm{CH}_{3}\right), 2.10 \sim 2.35$ $(\mathrm{m}, 2 \mathrm{H}, 17 \mathrm{a}-\mathrm{H}+17 \mathrm{~b}-\mathrm{H}), 2.46 \sim 2.60(\mathrm{~m}, 2 \mathrm{H}, 17 \mathrm{a}-\mathrm{H}+$ 17b-H), 3.08, 3.56, 3.68, 3.80 (each s, each $3 \mathrm{H}, \mathrm{CH}_{3}+$ $\left.\mathrm{OCH}_{3}\right), 3.64$ (q, $\left.J=7.6 \mathrm{~Hz}, 2 \mathrm{H}, 8 \mathrm{a}-\mathrm{H}\right), 4.16$ (q, $J=7.3 \mathrm{~Hz}$, $1 \mathrm{H}, 18-\mathrm{H}), 4.30$ (d, J=7.4 Hz, 1H, 17-H), 5.25 (s, 2H, 132-H), 9.42, 10.26 (each s, each 1H, 10-H), 11.34 (s, 1H, 3-CHO); IR (KBr) v: $3433(\mathrm{~N}-\mathrm{H}), 2973 \sim 2870(\mathrm{C}-\mathrm{H})$, $1735 \sim 1695(\mathrm{C}=\mathrm{O}), 1605(\mathrm{C}=\mathrm{C}), 1557$ (chlorin skeleton), 1440, 1326, 1172, 1013, $809 \mathrm{~cm}^{-1}$; MS m/z: 586.3 $(\mathrm{M}+2)^{+}$. Anal. calcd for $\mathrm{C}_{33} \mathrm{H}_{33} \mathrm{ClN}_{4} \mathrm{O}_{4}$ : C 67.74, H 5.68, N 9.58; found C 67.69, H 5.55, N 9.39.

3.3 Z-3b-苯基-20-氯焦脱镁叶绿酸-a 甲酯(3a)、E-3b苯基-20-氯焦脱镁叶绿酸-a 甲酯(3b)和 E-3b-苯基-132氧代-20-氯焦脱镁叶绿酸-a 甲酯(3c)的合成

将 $411 \mathrm{mg}$ 化合物 $2(0.703 \mathrm{mmol})$ 和 $411 \mathrm{mg}$ 氯化苠 基三苯基膦(1.054 mmol)溶解于 $120 \mathrm{~mL}$ 二氯甲烷中, 搅 拌下加入溶解于 $24 \mathrm{~mL}$ 水的 $80 \mathrm{mg}$ 氢氧化钠, 氮气保护, 室温下继续搅拌 $30 \mathrm{~min}$, 再先后向反应体系加入 $40 \mathrm{~mL}$ 冰水和 $40 \mathrm{~mL}$ 二氯甲烷，用 $2 \%$ 的盐酸溶液将混合物的 水层 $\mathrm{pH}$ 值调至 3 , 分出有机层, 水层用二氯甲烷萃取 $(40 \mathrm{~mL} \times 2)$, 合并有机相, 无水硫酸钠干燥，除去溶剂 后迅速用过量的重氮甲烷处理，加入乙酸中和未反应的 重氮甲烷，再减压除去溶剂，剩余物经柱层析分离[洗 脱剂: $V$ (石油醚)： $V$ (乙酸乙酯 $)=5 ： 1$ ], 分别得到 37 $\mathrm{mg}$ 墨绿色固体 3a $(0.056 \mathrm{mmol}, 8 \%), 121 \mathrm{mg}$ 橙色固体 $3 \mathbf{b}(0.183 \mathrm{mmol}, 26 \%)$ 和 $19 \mathrm{mg}$ 橙色固体 3c (0.028 mmol, $4 \%)$.

3a: m.p. $210 \sim 213{ }^{\circ} \mathrm{C}$; UV-vis $\left(\mathrm{CH}_{2} \mathrm{Cl}_{2}\right) \lambda_{\max }$

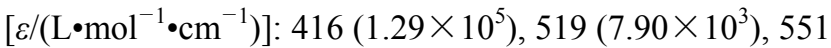
$\left(3.06 \times 10^{4}\right), 618\left(2.70 \times 10^{4}\right), 679\left(4.95 \times 10^{4}\right) \mathrm{nm} ;{ }^{1} \mathrm{H}$ NMR (400 MHz, $\left.\mathrm{CDCl}_{3}\right) \delta$ : -1.85 (br s, $\left.1 \mathrm{H}, \mathrm{NH}\right), 0.07$ (br s, 1H, NH), 1.67 (t, J=7.6 Hz, 3H, 8- $\mathrm{CH}_{3}$ ), 1.64 (d, $\left.J=8.1 \mathrm{~Hz}, 3 \mathrm{H}, 18-\mathrm{CH}_{3}\right), 2.00 \sim 2.06(\mathrm{~m}, 1 \mathrm{H}, 17 \mathrm{a}+$ $17 \mathrm{~b}-\mathrm{H}), 2.33 \sim 2.38(\mathrm{~m}, 1 \mathrm{H}, 17 \mathrm{a}+17 \mathrm{~b}-\mathrm{H}), 2.56 \sim 2.61(\mathrm{~m}$, $2 \mathrm{H}, 17 \mathrm{a}+17 \mathrm{~b}-\mathrm{H}$ ), 3.12, 3.35, 3.61, 3.69 (each s, each $3 \mathrm{H}$, $\mathrm{CH}_{3}+\mathrm{OCH}_{3}$ ), 3.67 (q, $\left.J=7.6 \mathrm{~Hz}, 2 \mathrm{H}, 8 \mathrm{a}-\mathrm{H}\right), 4.25$ (dd, $J=$ 9.2, $1.7 \mathrm{~Hz}, 1 \mathrm{H}, 17-\mathrm{H}), 4.80$ (q, $J=8.1 \mathrm{~Hz}, 1 \mathrm{H}, 18-\mathrm{H}$ ), $5.25\left(\mathrm{~d}, J=3.8 \mathrm{~Hz}, 2 \mathrm{H}, 13^{2}-\mathrm{H}\right), 6.98(\mathrm{dt}, J=8.0,3.2 \mathrm{~Hz}$, 
3H, PhH), 7.23 (d, $J=7.2 \mathrm{~Hz}, 2 \mathrm{H}, \mathrm{Ph}-\mathrm{H}), 7.49$ (d, $J=12.3$ Hz, 1H, 3b-H), 7.66 (d, $J=12.3 \mathrm{~Hz}, 1 \mathrm{H}, 3 \mathrm{a}-\mathrm{H}), 9.47,9.54$ (each s, each 1H, meso-H); IR (KBr) v: $3446(\mathrm{~N}-\mathrm{H}), 2924$ $(\mathrm{C}-\mathrm{H}), 1742 \sim 1697(\mathrm{C}=\mathrm{O}), 1654(\mathrm{C}=\mathrm{C}), 1560$ (chlorin skeleton), 1457, 1321, 1082, 1022, $974 \mathrm{~cm}^{-1}$; MS m/z: $660.4(\mathrm{M}+2)^{+}$. Anal. calcd for $\mathrm{C}_{40} \mathrm{H}_{39} \mathrm{ClN}_{4} \mathrm{O}_{3}: \mathrm{C} 72.88, \mathrm{H}$ 5.96, N 8.50; found C 72.69, H 6.14, N 8.42.

3b: m.p. $204 \sim 207{ }^{\circ} \mathrm{C}$; UV-vis $\left(\mathrm{CH}_{2} \mathrm{Cl}_{2}\right) \lambda_{\max }$

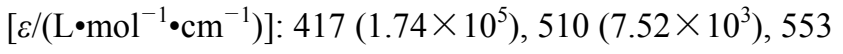
$\left.\left(2.90 \times 10^{4}\right), 662\left(2.57 \times 10^{4}\right), 6844.72 \times 10^{4}\right) \mathrm{nm} ;{ }^{1} \mathrm{H}$ NMR (400 MHz. $\mathrm{CDCl}_{3}$ ) $\delta$ : -1.87 (br s, $1 \mathrm{H}, \mathrm{NH}$ ), 0.64 (br s, $1 \mathrm{H}, \mathrm{NH}), 1.70$ (t, $\left.J=7.6 \mathrm{~Hz}, 3 \mathrm{H}, 8-\mathrm{CH}_{3}\right), 1.64$ (d, $\left.J=7.0 \mathrm{~Hz}, 3 \mathrm{H}, 18-\mathrm{CH}_{3}\right), 2.15 \sim 2.30(\mathrm{~m}, 2 \mathrm{H}, 17 \mathrm{a}+$ 17b-H), $2.66 \sim 2.50$ (m, 2H, $17 \mathrm{a}+17 \mathrm{~b}-\mathrm{H}), 3.22,3.60,3.65$, 3.69 (each s, each $3 \mathrm{H}, \mathrm{CH}_{3}+\mathrm{OCH}_{3}$ ), 3.69 (q, $J=7.6 \mathrm{~Hz}$, 2H, 8a-H), 4.24 (dd, $J=9.2,2.4 \mathrm{~Hz}, 1 \mathrm{H}, 17-\mathrm{H}), 4.82$ (q, $J=7.1 \mathrm{~Hz}, 1 \mathrm{H}, 18-\mathrm{H}), 5.21\left(\mathrm{~d}, J=20.0 \mathrm{~Hz}, 1 \mathrm{H}, 13^{2}-\mathrm{H}\right)$, $5.27\left(\mathrm{~d}, J=20.0 \mathrm{~Hz}, 1 \mathrm{H}, 13^{2}-\mathrm{H}\right), 7.48(\mathrm{t}, J=7.6 \mathrm{~Hz}, 1 \mathrm{H}$, Ph-H), 7.49 (d, $J=16.5 \mathrm{~Hz}, 1 \mathrm{H}, 3 \mathrm{~b}-\mathrm{H}), 7.58$ (t, $J=7.5 \mathrm{~Hz}$, 2H, Ph-H), 7.87 (d, $J=7.5$ H, 2H, Ph-H), 8.29 (d, $J=16.5$ $\mathrm{Hz}, 1 \mathrm{H}, 3 \mathrm{a}-\mathrm{H}$ ), 9.54, 9.60 (each s, each 1H, meso-H); IR (KBr) $v: 3441(\mathrm{~N}-\mathrm{H}), 2925(\mathrm{C}-\mathrm{H}), 1739 \sim 1690(\mathrm{C}=$ O), $1616(\mathrm{C}=\mathrm{N}), 1550$ (chlorin skeleton), 1462, 1367, 1224, 1166, 1036, $908 \mathrm{~cm}^{-1}$; MS m/z: $660.3(\mathrm{M}+2)^{+}$. Anal. calcd for $\mathrm{C}_{40} \mathrm{H}_{39} \mathrm{ClN}_{4} \mathrm{O}_{3}$ : C 72.88, H 5.96, N 8.50; found $\mathrm{C} 72.99, \mathrm{H} 5.79, \mathrm{~N} 8.61$.

3c: m.p. $217 \sim 220{ }^{\circ} \mathrm{C}$; UV-vis $\left(\mathrm{CH}_{2} \mathrm{Cl}_{2}\right) \lambda_{\max }$

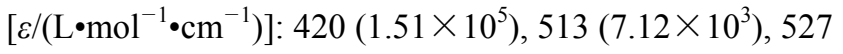
$\left(2.08 \times 10^{4}\right), 666\left(2.21 \times 10^{4}\right), 689\left(5.21 \times 10^{4}\right) \mathrm{nm} ;{ }^{1} \mathrm{H}$ NMR (400 MHz. $\left.\mathrm{CDCl}_{3}\right) \delta$ : $-2.26($ br s, $1 \mathrm{H}, \mathrm{NH}), 0.64$ (br s, $1 \mathrm{H}, \mathrm{NH}), 1.73$ (d, J=7.0 Hz, 3H, 18- $\left.\mathrm{CH}_{3}\right), 1.76$ (t, $\left.J=7.6 \mathrm{~Hz}, 3 \mathrm{H}, 8-\mathrm{CH}_{3}\right), 2.23 \sim 2.43(\mathrm{~m}, 2 \mathrm{H}, 17 \mathrm{a}+17 \mathrm{~b}-\mathrm{H})$, $2.55 \sim 2.75(\mathrm{~m}, 2 \mathrm{H}, 17 \mathrm{a}+17 \mathrm{~b}-\mathrm{H}), 3.60,3.65,3.71,3.87$ (each s, each $3 \mathrm{H}, \mathrm{CH}_{3}+\mathrm{OCH}_{3}$ ), 3.79 (q, $J=7.6 \mathrm{~Hz}, 2 \mathrm{H}$, $8 \mathrm{a}-\mathrm{H}$ ), 4.97 (q, $J=7.2 \mathrm{~Hz}, 1 \mathrm{H}, 18-\mathrm{H}), 5.24$ (dd, $J=8.6,3.2$ $\mathrm{Hz}, 1 \mathrm{H}, 17-\mathrm{H}), 7.49$ (t, $J=7.8 \mathrm{~Hz}, 1 \mathrm{H}, \mathrm{PhH}), 7.54$ (d, $J=$ $16.3 \mathrm{~Hz}, 1 \mathrm{H}, 3 \mathrm{~b}-\mathrm{H}), 7.60$ (t, $J=7.5 \mathrm{~Hz}, 2 \mathrm{H}, \mathrm{Ph}-\mathrm{H}), 7.91$ (d, $J=7.5 \mathrm{H}, 2 \mathrm{H}, \mathrm{Ph}-\mathrm{H}), 8.38$ (d, $J=16.3 \mathrm{~Hz}, 1 \mathrm{H}, 3 \mathrm{a}-\mathrm{H})$, 9.86, 10.02 (each s, each $1 \mathrm{H}$, meso-H); IR (KBr) v: 3344 $(\mathrm{N}-\mathrm{H}), 2862(\mathrm{C}-\mathrm{H}), 1749 \sim 1697(\mathrm{C}=\mathrm{O}), 1629(\mathrm{C}=\mathrm{C})$, 1591 (chlorin skeleton), 1494, 1261, 1082, $1041 \mathrm{~cm}^{-1}$. Anal. calcd for $\mathrm{C}_{40} \mathrm{H}_{37} \mathrm{ClN}_{4} \mathrm{O}_{4}$ : C 71.36, H 5.54, $\mathrm{N}$ 8.32; found C 71.50, H 5.41, N 8.55.
3.4 3-[5'( $R, S)$-(3'-对硝基苯基-4',5'-二氢异噁唑基)]-3去乙烯基 $-13^{2}$-氧代焦脱镁叶绿酸-a 甲酯 (5) 和 3-[5'(R,S)-(3'-对硝基苯基-4',5'-二氢异噁唑基)]-红紫 素-18 甲酯(6)的合成

在 $2 \mathrm{~mL}$ 干燥的二氯甲烷中溶解 $75 \mathrm{mg}$ NBS $(0.422$ $\mathrm{mmol})$, 一次性加入 $61 \mathrm{mg}$ 对硝基苯甲醛肜(0.280 $\mathrm{mmol}$ ), $20 \mathrm{~min}$ 后溴化反应结束, 再向反应体系加入 130 $\mathrm{mg}$ 二氢卟吩二酮 $4(0.231 \mathrm{mmol})$, 待其完全溶解后, 将 溶解于 $2 \mathrm{~mL}$ 氢氧化钠饱和甲醇溶液的 $80 \mathrm{mg}$ 三乙胺在 $15 \mathrm{~min}$ 之内滴入反应液. 氮气保护, 室温摚拌反应 $16 \mathrm{~h}$, 加入 $20 \mathrm{~mL}$ 二氯甲烷和 $30 \mathrm{~mL}$ 水分层, 分出有机相后用 水洗涤 $(20 \mathrm{~mL} \times 3)$, 减压浓缩, 将剩余物通过柱层析分 离混合物 [洗脱剂: $V$ (正己烷 $): V($ 乙酸乙酯 $)=3: 1$ ] 得 $35 \mathrm{mg}$ 红色固体 5 (0.049 mmol, 21\%)和 $16 \mathrm{mg}$ 红色固体 $6(0.021 \mathrm{mmol}, 10 \%)$. 5: m.p. $223 \sim 226{ }^{\circ} \mathrm{C}$; UV-vis

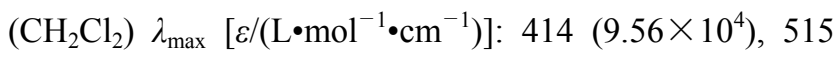
$\left(1.13 \times 10^{4}\right), \quad 548 \quad\left(6.69 \times 10^{3}\right), \quad 611 \quad\left(4.78 \times 10^{3}\right), \quad 669$ $\left(3.92 \times 10^{4}\right) \mathrm{nm} ;{ }^{1} \mathrm{H}$ NMR $\left(\mathrm{CDCl}_{3}\right) \delta:-1.89($ br s, $1 \mathrm{H}$, $\mathrm{NH}), 0.84$ (br s, 1H, NH), 1.68 (1.67) (t, $J=7.6 \mathrm{~Hz}, 3 \mathrm{H}$, $\left.8 \mathrm{a}-\mathrm{CH}_{3}\right), 1.87$ (d, $\left.J=7.1 \mathrm{~Hz}, 3 \mathrm{H}, 18-\mathrm{CH}_{3}\right), 2.84 \sim 2.62(\mathrm{~m}$, $2 \mathrm{H}, 17 \mathrm{a}+17 \mathrm{~b}-\mathrm{H}), 2.43 \sim 2.25$ (m, $2 \mathrm{H}, 17 \mathrm{a}+17 \mathrm{~b}-\mathrm{H}), 3.69$ (3.68) (q, $J=7.6 \mathrm{~Hz}, 2 \mathrm{H}, 8 \mathrm{a}-\mathrm{H}), 3.14,3.53$ (3.54), 3.59, 3.78 (3.76) (each s, each 3H, $\left.\mathrm{CH}_{3}+\mathrm{OCH}_{3}\right), 4.10$ (4.09) (d, $J=16.9 \mathrm{~Hz}, 1 \mathrm{H}, 3 \mathrm{~b}-\mathrm{H}), 4.36$ (4.38) (dd, $J=16.9,10.2 \mathrm{~Hz}$, 1H, 3b-H), 4.70 (q, $J=7.2 \mathrm{~Hz}, 1 \mathrm{H}, 18-\mathrm{H}$ ), 5.14 (d, $J=8.9$ Hz, 1H, 17-H), 7.23 (7.24) (t, $J=10.2 \mathrm{~Hz}, 1 \mathrm{H}, 3 \mathrm{a}-\mathrm{H}), 7.52$ (d, $J=8.5 \mathrm{~Hz}, 2 \mathrm{H}, \mathrm{PhH}), 7.89(7.88)(\mathrm{d}, J=8.5 \mathrm{~Hz}, 2 \mathrm{H}$, $\mathrm{PhH}), 9.00,9.75$ (9.72), 9.83 (9.82) (each $\mathrm{s}$, each $1 \mathrm{H}$, meso-H); IR (KBr) v: $3438(\mathrm{~N}-\mathrm{H}), 2956 \sim 2888(\mathrm{C}-\mathrm{H})$, $1701 \sim 1738(\mathrm{C}=\mathrm{O}), 1627(\mathrm{C}=\mathrm{C}) 1540$ (chlorin skeleton), 1519, 1401, 1074, $936 \mathrm{~cm}^{-1}$; MS $m / z: 727.7(\mathrm{M}+$ H) ${ }^{+}$. Anal. calcd for $\mathrm{C}_{41} \mathrm{H}_{38} \mathrm{~N}_{6} \mathrm{O}_{7}$ : C 67.76, H 5.27, N 11.56; found C 67.60, H 5.55, N 11.49. 化合物 6 的分析 数据与文献[16]一致.

$3.513^{2}$-氧代- $13^{1}$-邻氭基苯亚氨基- $13^{1}$-去甲基焦脱镁 叶绿酸-a 甲酯(7)的合成

将 $200 \mathrm{mg}$ MPPa (1) (0.364 mmol)溶解于 $15 \mathrm{~mL}$ 吡 啶中, $110{ }^{\circ} \mathrm{C}$ 避光摚拌下加入 $140 \mathrm{mg}$ 邻氨基苯甲腈 (0.001 mol), 三滴 TFA, TLC 监测, $8 \mathrm{~h}$ 后反应结束, 加入 $5 \mathrm{~mL}$ 浓盐酸淬灭反应, 再加入 $20 \mathrm{~mL}$ 二氯甲烷和水 20 $\mathrm{mL}$ 分层, 分出有机相, 水相用二氯甲烷萃取 (20 $\mathrm{mL} \times 3)$, 合并有机相, 用无水硫酸钠干燥, 减压蒸除溶 剂, 将剩余物通过柱层析分离混合物[洗脱剂: $V($ 正己 烷) $: V($ 乙酸乙酯 $)=4: 1$ ]得到 $113 \mathrm{mg}$ 红棕色固体 7 (0.171 mmol), 产率 47\%. m.p. $235 \sim 238{ }^{\circ} \mathrm{C}$; UV-vis 


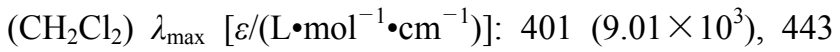
$\left(1.17 \times 10^{4}\right), \quad 526 \quad\left(1.52 \times 10^{3}\right), \quad 612\left(4.25 \times 10^{3}\right), \quad 676$ $\left(7.02 \times 10^{2}\right), 709\left(3.39 \times 10^{3}\right) \mathrm{nm} ;{ }^{1} \mathrm{H}$ NMR $(400 \mathrm{MHz}$, $\left.\mathrm{CDCl}_{3}\right) \delta:-2.19(-2.46)($ br s, $1 \mathrm{H}, \mathrm{NH}), 0.15(-0.03)$ (br s, 1H, NH), 1.73 (1.69) (t, $\left.J=7.6 \mathrm{~Hz}, 3 \mathrm{H}, 8-\mathrm{CH}_{3}\right), 1.90$ (d, $\left.J=7.3 \mathrm{~Hz}, 3 \mathrm{H}, 18-\mathrm{CH}_{3}\right), 2.26 \sim 2.36(\mathrm{~m}, 1 \mathrm{H}, 17 \mathrm{a}+$ 17b-H), $2.41 \sim 2.49$ (m, 1H, 17a+17b-H), 2.66 2.75 (m, $1 \mathrm{H}, 17 \mathrm{a}+17 \mathrm{~b}-\mathrm{H}), 2.98 \sim 3.04(\mathrm{~m}, 1 \mathrm{H}, 17 \mathrm{a}+17 \mathrm{~b}-\mathrm{H}), 3.31$, 3.50, 3.51 (3.53), 3.73 (each s, each $3 \mathrm{H}, \mathrm{CH}_{3}+\mathrm{OCH}_{3}$ ), 3.74 (q, $J=7.6 \mathrm{~Hz}, 2 \mathrm{H}, 8 \mathrm{a}-\mathrm{H}), 4.45(4.68)(\mathrm{q}, J=7.5 \mathrm{~Hz}$, $1 \mathrm{H}, 18-\mathrm{H}), 5.14(5.24)(\mathrm{d}, J=8.2 \mathrm{~Hz}, 1 \mathrm{H}, 17-\mathrm{H}), 6.24$ (6.22) (d, $J=11.5 \mathrm{~Hz}, 1 \mathrm{H}, 3 \mathrm{~b}-\mathrm{H}) 6.34$ (d, $J=17.9 \mathrm{~Hz}, 1 \mathrm{H}$, 3b-H), 7.29 (d, $J=7.6 \mathrm{~Hz}, 1 \mathrm{H}, \mathrm{PhH}), 7.37$ (d, $J=7.8 \mathrm{~Hz}$, $1 \mathrm{H}, \mathrm{PhH}), 7.68$ (t, $J=7.6 \mathrm{~Hz}, 1 \mathrm{H}, \mathrm{PhH}), 7.78$ (t, $J=7.8$ $\mathrm{Hz}, 1 \mathrm{H}, \mathrm{PhH}), 8.08$ (8.101) (dd, $J=17.9,11.5 \mathrm{~Hz}, 1 \mathrm{H}$, 3a-H), 8.92 (8.96), 9.69 (9.60), 9.74 (each s, each 1H, meso-H); IR (KBr) v: $3432(\mathrm{~N}-\mathrm{H}), 2921 \sim 2857(\mathrm{C}-\mathrm{H})$, $1740 \sim 1698(\mathrm{C}=\mathrm{O}), 1623(\mathrm{C}=\mathrm{C}), 1562$ (chlorin skeleton), 1461, 1373, 1220, 1161, 1027, $908 \mathrm{~cm}^{-1}$; MS m/z: $663.4(\mathrm{M}+\mathrm{H})^{+}$. Anal. calcd for $\mathrm{C}_{41} \mathrm{H}_{38} \mathrm{~N}_{6} \mathrm{O}_{3}: \mathrm{C} 74.30, \mathrm{H}$ 5.78, N 12.68; found C 74.39, H 5.68, N 12.92.

$3.6 E-3 b$ - 苯基- $13^{2}$-氧代焦脱镁叶绿酸-a 甲酯(9)、 $E$-3b- 苯基-(2,3-n)-喹喔啉焦脱镁叶绿酸甲酯(10)和 $E$-3b-苯基-(2,3-n)-苯并咪唑并红紫素-18 甲酯(11)的合 成

将 $866 \mathrm{mg}$ 化合物 $8(1.386 \mathrm{mmol})$ 溶解于 $280 \mathrm{~mL}$ 四 氢呋喃中, 再加入由 $\mathrm{LiOH}$ 饱和的 $10 \mathrm{~mL}$ 甲醇溶液, 室 温摚拌 $6 \mathrm{~h}$; 加入 $250 \mathrm{~mL}$ 冰水, 摚拌下滴加浓盐酸, 调 节 $\mathrm{pH}$ 为 $2 \sim 3$, 加入 $250 \mathrm{~mL}$ 二氯甲烷分层, 用二氯甲烷 萃取水相 $(40 \mathrm{~mL} \times 2)$, 合并有机层, 水洗, 用无水硫酸 钠干燥, 蒸馏除去溶剂, 再重新溶解于 $350 \mathrm{~mL}$ 含有 $5 \%$ 浓硫酸的甲醇溶液, 室温搅拌过夜. 先后加入 $300 \mathrm{~mL}$ 水和 $300 \mathrm{~mL}$ 二氯甲烷分层, 分出有机相并水洗两次, 干燥后除去溶剂, 将剩余物通过柱层析分离[洗脱剂: $V$ (正己烷) $: V$ (乙酸乙酯) $=3: 1$ ] 得 $248 \mathrm{mg}$ 黄色固体 9 (0.388 mmol, $28 \%)$. 然后, 将其溶解于 $25 \mathrm{~mL}$ 吡啶中, 加入 $0.3 \mathrm{~mL}$ 三氟乙酸和 $250 \mathrm{mg}$ 邻苯二胺 $(2.292 \mathrm{mmol})$, $50{ }^{\circ} \mathrm{C}$ 摚拌 $2 \mathrm{~h}$, 减压蒸出吡啶, 将所得浓缩物重新溶解 于 $30 \mathrm{~mL}$ 二氯甲烷中, 用水洗涤 $(30 \mathrm{~mL} \times 2)$, 干燥后浓 缩, 剩余物经硅胶柱层析分离[洗脱剂: $V$ (石油醚) : $V($ 乙酸乙酯 $)=9: 2]$, 分别得到 $46 \mathrm{mg}$ 黄色固体 10 (0.066 mmol, 17\%)和 $79 \mathrm{mg}$ 红色固体 11 (0.109 mol, $28 \%)$.

10: m.p. $213 \sim 216{ }^{\circ} \mathrm{C}$; UV-vis $\left(\mathrm{CHCl}_{3}\right) \lambda_{\max }$

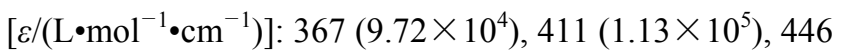

$\left.\left(9.27 \times 10^{4}\right), \quad 592 \quad\left(4.29 \times 10^{4}\right), \quad 654 \quad 2.03 \times 10^{4}\right), \quad 714$ $\left(6.55 \times 10^{4}\right) \mathrm{nm} ;{ }^{1} \mathrm{H}$ NMR $\left(400 \mathrm{MHz}, \mathrm{CDCl}_{3}\right) \delta:-0.90$ (br s, $1 \mathrm{H}, \mathrm{NH}$ ), 0.65 (br s, $1 \mathrm{H}, \mathrm{NH}), 1.87$ (d, $J=7.2 \mathrm{~Hz}$, $\left.3 \mathrm{H}, 18-\mathrm{CH}_{3}\right), 1.65\left(\mathrm{t}, J=7.6 \mathrm{~Hz}, 3 \mathrm{H}, 8 \mathrm{~b}-\mathrm{CH}_{3}\right), 2.28 \sim 2.51$ (m, 2H, 17a $+17 \mathrm{~b}-\mathrm{H}), 2.72 \sim 2.80(\mathrm{~m}, 1 \mathrm{H}, 17 \mathrm{a}+17 \mathrm{~b}-\mathrm{H})$, $2.88 \sim 2.97$ (m, 1H, 17a +17b-H), 3.57 (q, $J=7.6 \mathrm{~Hz}, 2 \mathrm{H}$, $8 \mathrm{a}-\mathrm{H}$ ), 3.15, 3.41, 3.59, 3.67 (each s, each $3 \mathrm{H}, \mathrm{OCH}_{3}+$ $\left.\mathrm{CH}_{3}\right), 4.51(\mathrm{q}, J=7.3 \mathrm{~Hz}, 1 \mathrm{H}, 18-\mathrm{H}), 5.10(\mathrm{~d}, J=8.1 \mathrm{~Hz}$, $17-\mathrm{H}), 7.45(\mathrm{t}, J=7.4 \mathrm{~Hz}, 1 \mathrm{H}, \mathrm{PhH}), 7.53 \sim 7.59(\mathrm{~m}, 4 \mathrm{H}$, $\mathrm{PhH}), 7.57$ (d, $J=16.4 \mathrm{~Hz}, 3 \mathrm{~b}-\mathrm{H}), 7.85(\mathrm{~d}, J=7.5 \mathrm{~Hz}, 2 \mathrm{H}$, ArH), 8.04 (dd, $J=7.3,2.0 \mathrm{~Hz}, 3 \mathrm{H}, \mathrm{ArH}), 8.29$ (d, $J=16.4$ $\mathrm{Hz}, 3 \mathrm{a}-\mathrm{H}), 8.34 \sim 8.62(\mathrm{~m}, 2 \mathrm{H}, \mathrm{PhH}), 8.56,9.30,9.42$ (each s, each 1H, meso-H); IR (KBr) v: $3440(\mathrm{~N}-\mathrm{H}), 2929$ $(\mathrm{C}-\mathrm{H}), 1741 \sim 1690(\mathrm{C}=\mathrm{O}), 1662(\mathrm{C}=\mathrm{C}), 1502$ (chlorin skeleton), 1258, 1093, 1022, $806 \mathrm{~cm}^{-1}$; MS m/z: 711.4 $(\mathrm{M}+\mathrm{H})^{+}$. Anal. calcd for $\mathrm{C}_{46} \mathrm{H}_{42} \mathrm{~N}_{5} \mathrm{O}_{2}$ : C 77.72, H 5.96, N 11.82; found C 77.54, H 5.90, N 11.69 .

11: m.p. $222 \sim 225{ }^{\circ} \mathrm{C}$; UV-vis $\left(\mathrm{CHCl}_{3}\right) \lambda_{\max }$

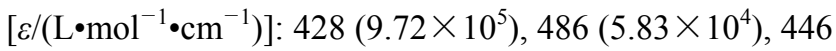
$\left(5.83 \times 10^{4}\right), \quad 556 \quad\left(2.72 \times 10^{4}\right), \quad 670 \quad\left(7.78 \times 10^{3}\right), \quad 730$ $\left(4.86 \times 10^{4}\right) \mathrm{nm} ;{ }^{1} \mathrm{H}$ NMR $\left(400 \mathrm{MHz}, \mathrm{CDCl}_{3}\right) \delta: 0.28(\mathrm{br} \mathrm{s}$, $1 \mathrm{H}, \mathrm{NH}), 0.56$ (br s, $1 \mathrm{H}, \mathrm{NH}), 1.81(\mathrm{~d}, J=7.2 \mathrm{~Hz}, 3 \mathrm{H}$, $\left.18-\mathrm{CH}_{3}\right), 1.61\left(\mathrm{t}, J=7.6 \mathrm{~Hz}, 3 \mathrm{H}, 8 \mathrm{~b}-\mathrm{CH}_{3}\right), 2.01 \sim 2.10(\mathrm{~m}$, $1 \mathrm{H}, \quad 17 \mathrm{a}+17 \mathrm{~b}-\mathrm{H}), \quad 2.27 \sim 2.50(\mathrm{~m}, 2 \mathrm{H}, 17 \mathrm{a}+17 \mathrm{~b}-\mathrm{H})$, $2.75 \sim 2.81(\mathrm{~m}, 1 \mathrm{H}, 17 \mathrm{a}+17 \mathrm{~b}-\mathrm{H}), 3.52(\mathrm{q}, J=7.6 \mathrm{~Hz}, 2 \mathrm{H}$, $8 \mathrm{a}-\mathrm{H}$ ), 3.04, 3.32, 3.61, 3.66 (each s, each $3 \mathrm{H}, \mathrm{OCH}_{3}+$ $\mathrm{CH}_{3}$ ), 4.37 (q, $\left.J=7.3 \mathrm{~Hz}, 1 \mathrm{H}, 18-\mathrm{H}\right), 5.40$ (dd, $J=8.5,1.7$ $\mathrm{Hz}, 17-\mathrm{H}), 7.42 \sim 7.47$ (m, 3H, PhH), $7.53(\mathrm{~d}, J=16.7 \mathrm{~Hz}$, $1 \mathrm{H}, 3 \mathrm{a}-\mathrm{H})), 7.55$ (t, $J=7.5 \mathrm{~Hz}, 2 \mathrm{H}, \mathrm{PhH}), 7.79$ (d, $J=7.5$ $\mathrm{Hz}, 2 \mathrm{H}, \mathrm{ArH}), 7.93$ (t, $J=7.5 \mathrm{~Hz}, 1 \mathrm{H}, \mathrm{ArH}), 8.08$ (d, $J=$ $16.7 \mathrm{~Hz}, 1 \mathrm{H}, 3 \mathrm{a}-\mathrm{H}), 8.75$ (dd, $J=7.5,2.8 \mathrm{~Hz}, 1 \mathrm{H}, \mathrm{ArH})$, 8.54, 9.20, 9.31 (each s, each 1H, meso-H); IR (KBr) v: $3432(\mathrm{~N}-\mathrm{H}), 2924,2860(\mathrm{C}-\mathrm{H}), 1738 \sim 1682(\mathrm{C}=\mathrm{O})$, $1637(\mathrm{C}=\mathrm{C}), 1561$ (chlorin skeleton), 1508, 1400, 1082 $\mathrm{cm}^{-1}$. MS m/z: $727.4(\mathrm{M}+\mathrm{H})^{+}$. Anal. calcd for $\mathrm{C}_{46} \mathrm{H}_{42} \mathrm{~N}_{6} \mathrm{O}_{3}: \mathrm{C} 76.01, \mathrm{H} 5.82, \mathrm{~N} 11.56$; found $\mathrm{C} 76.10, \mathrm{H}$ $6.00, \mathrm{~N} 11.70$.

\subsection{2-(2-氨基-4/5-苯甲酰苯亚氨基)-12-去甲基焦脱 镁叶绿酸-a 甲酯(13)的合成}

将 $70 \mathrm{mg}$ 12-甲酰基焦脱镁叶绿酸-a 甲酯 $\mathbf{1 2}(0.124$

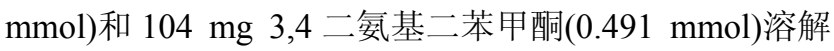
于 $5 \mathrm{~mL}$ 吡啶中, 加入 $0.1 \mathrm{~mL}$ 三氟乙酸, $50{ }^{\circ} \mathrm{C}$ 避光搅拌 反应 $7 \mathrm{~h}$, 减压蒸除吡啶, 将所得浓缩物重新溶解于 30 $\mathrm{mL}$ 二氯甲烷中, 用水洗涤 $(30 \mathrm{~mL} \times 2)$, 干燥后浓缩, 所 得浓缩物经硅胶柱层析分离[洗脱剂: $V$ (石油醚) $: V($ 乙 
酸乙酯 $)=2: 1$ ], 得到 $36 \mathrm{mg}$ 黄色固体 $13(0.047 \mathrm{mmol})$, 产率 38\%. m.p. 225 228 ${ }^{\circ} \mathrm{C}$; UV-vis $\left(\mathrm{CH}_{2} \mathrm{Cl}_{2}\right) \lambda_{\max }$

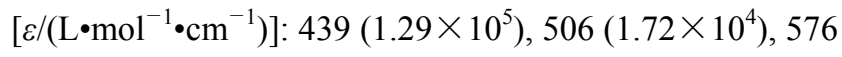
$\left(3.52 \times 10^{3}\right), 634\left(2.71 \times 10^{4}\right), 693\left(7.74 \times 10^{4}\right) \mathrm{nm} ;{ }^{1} \mathrm{H}$ $\operatorname{NMR}\left(\mathrm{CDCl}_{3}\right) \delta$ : $-0.85($ br s, $1 \mathrm{H}, \mathrm{NH}),-0.58(-0.55)$ (br s, 1H, NH), $1.52(1.56)\left(\mathrm{t}, J=7.4 \mathrm{~Hz}, 3 \mathrm{H}, 8-\mathrm{CH}_{3}\right), 1.88$ (1.87) (d, $\left.J=7.3 \mathrm{~Hz}, 3 \mathrm{H}, 18-\mathrm{CH}_{3}\right), 2.34 \sim 2.49(\mathrm{~m}, 2 \mathrm{H}$, $17 \mathrm{a}+17 \mathrm{~b}-\mathrm{H}), 2.57 \sim 2.77(\mathrm{~m}, 2 \mathrm{H}, 17 \mathrm{a}+17 \mathrm{~b}-\mathrm{H}), 3.43 \sim$ 3.55 (m, 2H, 8a-H), 3.06 (3.08), 3.17 (3.10), 3.69 (3.68) (each s, each $\left.3 \mathrm{H}, \mathrm{OCH}_{3}+\mathrm{CH}_{3}\right), 3.98 \sim 4.08(\mathrm{~m}, 17-\mathrm{H})$, $4.17 \sim 4.26$ (m, 1H, 18-H), 4.90 (4.62) (d, $J=19.6 \mathrm{~Hz}, 1 \mathrm{H}$, $\left.13^{2}-\mathrm{H}\right), 4.83(4.53)\left(\mathrm{d}, J=19.6 \mathrm{~Hz}, 1 \mathrm{H}, 13^{2}-\mathrm{H}\right), 6.10(6.12)$ (dd, $J=11.5,1.0,1 \mathrm{H}, 3 \mathrm{~b}-\mathrm{H}), 6.17$ (6.20) (dd, $J=17.9,1.0$, $1 \mathrm{H}, 3 \mathrm{~b}-\mathrm{H}$ ), $7.48 \sim 7.79$ (m, 5H, Ph-H), $7.89 \sim 8.00$ (m, 5H, meso-H+3a-H+Ph-H), 8.82 (8.83), 9.79 (9.81) (each s, each 1H, meso-H), $11.93(11.81)(\mathrm{s}, 1 \mathrm{H}, 12 \mathrm{a}-\mathrm{H})$; IR $(\mathrm{KBr})$ v: $3454(\mathrm{~N}-\mathrm{H}), 2925(\mathrm{C}-\mathrm{H}), 1739 \sim 1687(\mathrm{C}=\mathrm{O}), 1654$, $1618(\mathrm{C}=\mathrm{C}), 1561$ (chlorin skeleton), 1448, 1380, 1253, 1172, 1083, 1010, 800, $729 \mathrm{~cm}^{-1}$; MS $m / z: 653.3(\mathrm{M}+$ $\mathrm{H})^{+}$. Anal. calcd for $\mathrm{C}_{47} \mathrm{H}_{44} \mathrm{~N}_{6} \mathrm{O}_{4}$ : C 74.58, H 5.86, N 11.10; found C 74.41, H 6.03, N 11.20.

\section{$3.8 \mathrm{~N}$-对氨基苄基-红紫素-18 亚酰胺甲酯(15)的合成}

将 $200 \mathrm{mg}$ 红紫素-18 甲酯 $\mathbf{1 4}(0.345 \mathrm{mmol})$ 溶解于 $15 \mathrm{~mL}$ 甲苯中, 加入 $200 \mathrm{mg}$ 对氨基市胺, 油浴 $120^{\circ} \mathrm{C}$ 摚 拌回流, TLC 检测反应进程, $7 \mathrm{~h}$ 反应完毕, 减压除去溶 剂, 将浓缩物再溶解于 $30 \mathrm{~mL}$ 二氯甲烷中, 用水洗涤 (30 $\mathrm{mL} \times 2$ ), 干燥后浓缩, 剩余物经硅胶柱层析分离[洗脱 剂: $V$ (石油醚) $: V$ (乙酸乙酯 $)=3: 1$ ], 得 $168 \mathrm{mg}$ 红色固 体 15 (0.245 mmol), 产率 $71 \%$. m.p. $231 \sim 233{ }^{\circ} \mathrm{C}$;

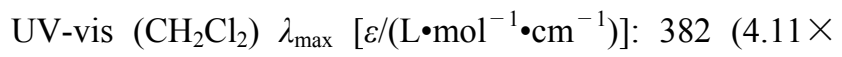
$\left.10^{4}\right), 437\left(9.78 \times 10^{4}\right), 530\left(5.87 \times 10^{3}\right), 569\left(1.76 \times 10^{4}\right)$, $706\left(3.42 \times 10^{4}\right) \mathrm{nm} ;{ }^{1} \mathrm{H}$ NMR $\left(\mathrm{CDCl}_{3}\right) \delta$ : $-0.21(\mathrm{br} \mathrm{s}$, $1 \mathrm{H}, \mathrm{NH}),-0.12(\mathrm{br} \mathrm{s}, 1 \mathrm{H}, \mathrm{NH}), 1.64$ (t, $J=7.6 \mathrm{~Hz}, 3 \mathrm{H}$, $\left.8-\mathrm{CH}_{3}\right), 1.75\left(\mathrm{~d}, J=7.3 \mathrm{~Hz}, 3 \mathrm{H}, 18-\mathrm{CH}_{3}\right), 1.92 \sim 2.08(\mathrm{~m}$, $1 \mathrm{H}, \quad 17 \mathrm{a}+17 \mathrm{~b}-\mathrm{H}), \quad 2.32 \sim 2.48(\mathrm{~m}, 2 \mathrm{H}, 17 \mathrm{a}+17 \mathrm{~b}-\mathrm{H})$, $2.63 \sim 2.73(\mathrm{~m}, 1 \mathrm{H}, 17 \mathrm{a}+17 \mathrm{~b}-\mathrm{H}), 3.13,3.34,3.58,3.80$ (each s, each $\left.3 \mathrm{H}, \mathrm{CH}_{3}+\mathrm{OCH}_{3}\right), 3.63(\mathrm{q}, J=7.6 \mathrm{~Hz}, 2 \mathrm{H}$, $8 \mathrm{a}-\mathrm{H}), 4.35$ (q, $J=7.3 \mathrm{~Hz}, 1 \mathrm{H}, 18-\mathrm{H}), 5.41$ (dd, $J=8.4,2.9$ $\mathrm{Hz}, 1 \mathrm{H}, 17-\mathrm{H}), 5.52$ (br, 2H, $\mathrm{PhNH}_{2}$ ), 5.54 (d, $J=14.0 \mathrm{~Hz}$, $1 \mathrm{H}, \mathrm{NCH}_{2} \mathrm{Ph}$ ), 5.64 (d, $\left.J=14.0 \mathrm{~Hz}, 1 \mathrm{H}, \mathrm{NCH}_{2} \mathrm{Ph}\right), 6.15$ (dd, $J=11.5,1.0 \mathrm{~Hz}, 1 \mathrm{H}, 3 \mathrm{~b}-\mathrm{H}$ ), 6.27 (dd, $J=17.9,1.0 \mathrm{~Hz}$, $1 \mathrm{H}, 3 \mathrm{~b}-\mathrm{H}), 6.70$ (d, $J=8.4 \mathrm{~Hz}, 2 \mathrm{H}, \mathrm{PhH}), 7.62$ (d, $J=8.4$ $\mathrm{Hz}, 2 \mathrm{H}, \mathrm{PhH}), 7.88$ (dd, $J=17.9,11.5 \mathrm{~Hz}, 1 \mathrm{H}, 3 \mathrm{a}-\mathrm{H}), 8.56$, 9.33, 9.57 (each s, each $1 \mathrm{H}$, meso-H); IR $(\mathrm{KBr}) v: 3449$ $(\mathrm{N}-\mathrm{H}), 2924(\mathrm{C}-\mathrm{H}), 1740 \sim 1689(\mathrm{C}=\mathrm{O}), 1654(\mathrm{C}=\mathrm{C})$,
1535 (chlorin skeleton), 1458, 1400, 1073, $883 \mathrm{~cm}^{-1}$; MS m/z: $683.4(\mathrm{M}+\mathrm{H})^{+}$. Anal. calcd for $\mathrm{C}_{41} \mathrm{H}_{42} \mathrm{~N}_{6} \mathrm{O}_{4}: \mathrm{C} 72.12$, H 6.20, N 12.31; found C 72.03, H 6.33, N 12.19.

\section{$3.9 \mathrm{~N}$-对甲酰苯甲叉氨基-红紫素-18 亚酰胺甲酯(16a) 的合成}

将 $189 \mathrm{mg}$ 红紫素-18 14 (0.326 mmol)溶解于 $50 \mathrm{~mL}$ 二氯甲烷中, 在氮气保护下加入 $0.3 \mathrm{~mL} 80 \%$ 的水合联 氨和 $0.2 \mathrm{~mL}$ 三乙胺, 室温搅拌 $4 \mathrm{~h}$, 水洗 $(30 \mathrm{~mL} \times 3)$, 有 机相用无水硫酸钠干燥, 减压浓缩至干; 用 $25 \mathrm{~mL}$ 二氯 甲烷溶解所得浓缩物, 搅拌下滴加含有 $80 \mathrm{mg}$ 对苯二甲 醛的 $2 \mathrm{~mL}$ 二氯甲烷溶液, 再加入 $15 \mathrm{mg}$ 对甲苯磺酸, 室 温搅拌反应 $12 \mathrm{~h}$, 水洗 $(80 \mathrm{~mL} \times 3)$, 用无水硫酸钠干燥 后减压浓缩, 所得浓缩物经硅胶柱层析分离[洗脱剂: $V$ (石油醚) $: V($ 乙酸乙酯 $)=3: 2]$, 得到 $97 \mathrm{mg}$ 紫红色产 物 16a $(0.137 \mathrm{mmol})$, 产率 $42 \%$. m.p. $217 \sim 220{ }^{\circ} \mathrm{C}$;

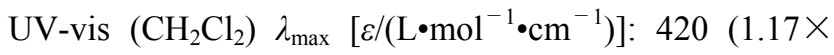
$\left.10^{5}\right), 522\left(5.85 \times 10^{3}\right), 552\left(9.36 \times 10^{3}\right), 649\left(9.36 \times 10^{3}\right)$, $716\left(3.74 \times 10^{4}\right) \mathrm{nm} ;{ }^{1} \mathrm{H}$ NMR $\left(\mathrm{CDCl}_{3}\right) \delta: 0.19(\mathrm{br} \mathrm{s}, 1 \mathrm{H}$, $\mathrm{NH}), 0.06$ (br s, 1H, NH), 1.59 (t, J=7.6 Hz, 3H, 8- $\mathrm{CH}_{3}$ ), $1.61\left(\mathrm{~d}, J=7.3 \mathrm{~Hz}, 3 \mathrm{H}, 18-\mathrm{CH}_{3}\right), 1.96 \sim 2.02(\mathrm{~m}, 1 \mathrm{H}$, $17 \mathrm{a}+17 \mathrm{~b}-\mathrm{H}), 2.38 \sim 2.48(\mathrm{~m}, 2 \mathrm{H}, 17 \mathrm{a}+17 \mathrm{~b}-\mathrm{H}), 2.71 \sim$ $2.78(\mathrm{~m}, 1 \mathrm{H}, 17 \mathrm{a}+17 \mathrm{~b}-\mathrm{H}), 3.08,3.32,3.52,3.71$ (each s, each $\left.3 \mathrm{H}, \mathrm{CH}_{3}+\mathrm{OCH}_{3}\right), 3.54$ (q, $\left.J=7.6 \mathrm{~Hz}, 2 \mathrm{H}, 8 \mathrm{a}-\mathrm{H}\right)$, 4.33 (q, $J=7.3 \mathrm{~Hz}, 1 \mathrm{H}, 18-\mathrm{H}), 5.29$ (dd, $J=8.4,2.9 \mathrm{~Hz}$, $1 \mathrm{H}, 17-\mathrm{H}), 6.14$ (dd, $J=11.5,1.0 \mathrm{~Hz}, 1 \mathrm{H}, 3 \mathrm{~b}-\mathrm{H}), 6.27$ (dd, $J=17.9,1.0 \mathrm{~Hz}, 1 \mathrm{H}, 3 \mathrm{~b}-\mathrm{H}), 7.84$ (d, $J=8.4 \mathrm{~Hz}, 2 \mathrm{H}, \mathrm{PhH}$ ), $8.14(\mathrm{~d}, J=8.4 \mathrm{~Hz}, 2 \mathrm{H}, \mathrm{PhH}), 7.88(\mathrm{dd}, J=17.9,11.5 \mathrm{~Hz}$, $1 \mathrm{H}, 3 \mathrm{a}-\mathrm{H}$ ), 8.05, 9.02, 9.24 (each s, each $1 \mathrm{H}$, meso-H), 9.44 (s, 1H, N=CH), 10.14 (s, 1H, PhCHO); IR (KBr) v: $3421(\mathrm{~N}-\mathrm{H}), 2958(\mathrm{C}-\mathrm{H}), 1739 \sim 1689(\mathrm{C}=\mathrm{O}), 1612$ $(\mathrm{C}=\mathrm{C}) 1552$ (chlorin skeleton), 1463, 1375, 1259, 1211, $1114 \mathrm{~cm}^{-1}$; MS m/z: $709.4(\mathrm{M}+\mathrm{H})^{+}$. Anal. calcd for $\mathrm{C}_{42} \mathrm{H}_{40} \mathrm{~N}_{6} \mathrm{O}_{5}$ : C 71.17, H 5.69, N 11.86; found C 71.29, H 5.80, N 11.68 .

\section{$3.10 \mathrm{~N}$-苯甲酰氧基-红紫素-18 亚酰胺甲酯(16b)的合 成}

在 $15 \mathrm{~mL}$ 吡啶中溶解 $120 \mathrm{mg}$ 化合物 $\mathbf{1 4}(0.207$ $\mathrm{mmol})$, 加入 $350 \mathrm{mg}$ 研细的盐酸差弪胺, 室温下搅拌反应 $5 \mathrm{~h}$, 再加入 $5 \%$ 的盐酸水溶液 $200 \mathrm{~mL}$, 用二氯甲烷萃取 $(80 \mathrm{~mL} \times 3)$, 水洗有机相除酸, 用无水硫酸钠干燥, 减 压除尽溶剂, 将剩余物重新溶解于 $3 \mathrm{~mL}$ 吡啶中, 缓慢 滴加 $0.2 \mathrm{~mL}$ 苯甲酰氯, 室温摚拌, TLC 检测反应进程, 1 $\mathrm{h}$ 反应结束, 加入 $30 \mathrm{~mL}$ 二氯甲烷和 $30 \mathrm{~mL}$ 水分层, 水 相用二氯甲烷萃取 $(20 \mathrm{~mL} \times 3)$, 合并有机相, 再用 100 $\mathrm{mL}$ 水洗涤两次, 无水硫酸钠干燥, 减压蒸除溶剂, 所得 
浓缩物经硅胶柱层析分离 [洗脱剂: $V$ (石油醚)： $V($ 乙酸 乙酯 $)=1$ : 2], 得到 $77 \mathrm{mg}$ 紫红色产物 $16 \mathbf{b}(0.110$ mmol), 产率 53\%. m.p. 210 $214{ }^{\circ} \mathrm{C}$; UV-vis $\left(\mathrm{CH}_{2} \mathrm{Cl}_{2}\right)$

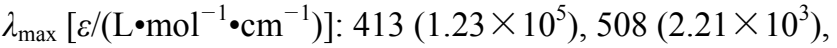
$545\left(6.15 \times 10^{4}\right), 645\left(2.46 \times 10^{4}\right), 711\left(8.17 \times 10^{4}\right) \mathrm{nm} ;{ }^{1} \mathrm{H}$ NMR $\left(\mathrm{CDCl}_{3}\right) \delta: 0.10$ (br s, 1H, NH), 0.18 (br s, 1H, NH), $1.55\left(\mathrm{t}, J=7.6 \mathrm{~Hz}, 3 \mathrm{H}, 8-\mathrm{CH}_{3}\right), 1.71(\mathrm{~d}, J=6.3 \mathrm{~Hz}, 3 \mathrm{H}$, $\left.18-\mathrm{CH}_{3}\right), 1.90 \sim 2.05(\mathrm{~m}, 1 \mathrm{H}, 17 \mathrm{a}+17 \mathrm{~b}-\mathrm{H}), 2.37 \sim 2.51$ (m, 2H, 17a+17b-H), 2.64 $2.82(\mathrm{~m}, 1 \mathrm{H}, 17 \mathrm{a}+17 \mathrm{~b}-\mathrm{H})$, 2.98, 3.27, 3.55 (3.53), 3.69 (3.68) (each s, each $3 \mathrm{H}$, $\mathrm{CH}_{3}+\mathrm{OCH}_{3}$ ), 3.42 (q, $\left.J=7.6 \mathrm{~Hz}, 2 \mathrm{H}, 8 \mathrm{a}-\mathrm{H}\right), 4.33$ (q, $J=$ $7.2 \mathrm{~Hz}, 1 \mathrm{H}, 18-\mathrm{H}), 5.28$ (5.20) (d, $J=8.4 \mathrm{~Hz}, 1 \mathrm{H}, 17-\mathrm{H})$, $6.09(\mathrm{~d}, J=11.5,1.0 \mathrm{~Hz}, 1 \mathrm{H}, c i s-3 \mathrm{~b}-\mathrm{H}), 6.20$ (d, $J=17.8$ $\mathrm{Hz}, 1 \mathrm{H}$, trans-3b-H), 7.62 (t, $J=7.6 \mathrm{~Hz}, 2 \mathrm{H}, \mathrm{PhH}), 7.74$ (dd, $J=17.8,11.5 \mathrm{~Hz}, 3 \mathrm{a}-\mathrm{H}), 7.73$ (d, $J=7.2 \mathrm{~Hz}, 1 \mathrm{H}$, Ph-H), 8.46 (d, J=7.6 Hz, 2H, Ph-H), 8.49, 9.50, 9.10, 9.32 (each s, each $1 \mathrm{H}$, meso-H); IR (KBr) v: $3446(\mathrm{~N}-\mathrm{H})$, 2960, 2927, $2858(\mathrm{C}-\mathrm{H}), 1737,1704(\mathrm{C}=\mathrm{O}), 1674(\mathrm{C}=$ C), 1542 (chlorin skeleton), 1523, 1458, 1398, 1174, 1068, $1051 \mathrm{~cm}^{-1}$; MS m/z: $689.3(\mathrm{M}+\mathrm{H})^{+}$. Anal. calcd for $\mathrm{C}_{41} \mathrm{H}_{39} \mathrm{~N}_{5} \mathrm{O}_{6}$ : C 70.57, H 5.63, N 10.04; found $\mathrm{C} \mathrm{70.79,} \mathrm{H}$ 5.69, N 10.22.

\section{References}

[1] (a) Gil, M.; Bieniaszl, M.; Seshadri, M.; Fisher, D.; Ciesielski, M. J.; Chen, Y.; Pandey, R. K.; Kozbor, D. Brit. J. Cancer 2011, 103, 1.

(b) Bellnier, D. A.; Greco, W. R.; Loewen, G. M.; Nava, H.; Oseroff, A. R.; Pandey, R. K.; Tsuchida, T.; Dougherty, T. J. Cancer Res. 2003, 63, 1806.

[2] (a) Tamiaki, H.; Shibata, R.; Mizoguchi, T. Photochem. Photobiol. 2007, 83, 152

(b) Goswami, L. N.; Ethirajan, M.; Dobhal, M. P.; Zhang, M.; Missert, J. R.; Shibata, M.; Kadish, K. M.; Pandey, R. K. J. Org. Chem. 2009, 74, 568

[3] Hoober, J. K.; Eggink, L. L.; Chen, M. Photosynth. Res. 2007, 94, 387.

[4] (a) Ethirajan, M.; Joshi, P.; William, W. H.; Ohkubo, K.; Fukuzumi, S.; Pandey, R. K. Org. Lett. 2011, 8, 1956.

(b) Mennenga, A.; Wolfgang Gartner, W.; Lubitz, W.; Gorner, H. Phys. Chem. Chem. Phys. 2006, 8, 5444.

(c) Ethirajan, M.; Joshi, P.; William, W. H.; Ohkubo, K.; Fukuzumi, S.; Pandey, R. K. Org. Lett, 2011, 13, 1956.

[5] Chen, Y. H.; Li, G. L.; Pandey, R. K. Curr. Org. Chem. 2004, 8, 1105.

[6] (a) Washington, I.; Brooks, C.; Turro, N. J.; Nakanishi, K. J. Am.
Chem. Soc. 2004, 126, 9892

(b). Steruberg, E. D.; Dolphin, D. Tetrahedron 1998, 54, 415.

(c) Zheng, G.; Potter, W. R.; Camacho, S. H.; Missert, J. R.; Wang, G.-S.; Bellnier, D. A.; Henderson, B. W.; Rodgers, M. A. J.; Dougherty, T. J.; Pandey, R. K. J. Med. Chem. 2001, 44, 1540.

[7] (a) Wang, J. J.; Li, J.-J.; Wu, X.-R.; Shim, Y.-K. Chin. J. Chem. 2006, 24, 933.

(b) Wang, J.-J.; Li, J.-Z.; Li, Y.-W.; Jakus, J.; Shim, Y.-K. J. Porphyrins Phthalocyanines 2010, 14, 859.

(c) Ji, J.-Y.; Li, J.-Z.; Wang, H.; Li, F.-G.; Han, G.-F.; Shen, R.-K.; Wang, J.-J. Chin. J. Org. Chem. 2006, 26, 1714 (in Chinese).

(纪建业, 李家柱, 王虎, 李付国, 韩光范, 沈荣基, 王进军, 有 机化学, 2006, 26, 1714.)

(d) Li, J.-Z.; Wang, J.-J.; Yoon, L.; Cui, B.-C.; Shim, Y.-K. Bioorg. Med. Chem. Lett. 2012, 22, 1846.

(e) Wang, J. J.; Liu, C.-L.; Li, J.-J. Synth. Commun. 2012, 42, 487.

(f) Wang, L.-M.; Wang, Z.; Yang. Z.; Jin, Y.-X.; Wang, J.-J. Chin. J. Org. Chem. 2012, 32, 2154 (in Chinese).

(王鲁敏, 王振, 杨泽, 金英学, 王进军, 有机化学, 2012, 32, 2154.)

(g) Wang, J.-J.; Li, F.-G.; Li, Y.-W. Chin. J. Org. Chem. 2011, 31, 68 (in Chinese).

(王进军, 李付国, 李韵伟, 有机化学, 2011, 31, 68.)

[8] Wang, J.-J. Chin. J. Org. Chem. 2005, 25, 1353 (in Chinese). (王进军, 有机化学, 2005, 25, 1353.)

[9] Wang, J.-J.; Wu, X.-R.; Wang, L.-M.; Han, G.-F.; Shin, R.-K. Chin. J. Org. Chem. 2004, 24, 906 (in Chinese).

(王进军, 邬旭然, 王鲁敏, 韩光范, 沈荣基, 有机化学, 2004, 24, 906.)

[10] (a) Li, J.-J.; Liu, W.-H.; Li, F.-G.; Wang, J.-J.; Suo, Y.-R.; Liu, Y.-J. Chin. J. Org. Chem. 2007, 27, 1594 (in Chinese).

(李家柱, 刘万卉, 李付国, 王进军, 索有瑞, 刘永军, 有机化 学, 2007, 27, 1594.)

(b) Tamoata, H.; Kouraba, M. Tetrahedron 1997, 53, 10677.

[11] (a) Li, J.-Z.; Wang, J.-J.; Yoon, L.; Cui, B.-C.; Shim, Y.-K. Bioorg. Med. Chem. Lett. 2012, 22, 1846.

(b) Wang, J.J.; Liu, C.-L.; Li, J.-J. Synth. Commun. 2012, 42, 487.

(c) Wang, J.-J.; Wang, P.; Li, J.-J.; Jakus, J.; Shim, Y.-K. Bull. Korean Chem. Soc. 2011, 32, 3473.

[12] Li, J.-Z. M.S. Thesis, Yantai University, Yantai, 2007 (in Chinese). (李家柱，硕士论文，烟台大学，烟台, 2007.)

[13] Wu, X.-R.; Liu, C.; Yang, Z.; Yao, N.-N.; Wang, J.-J. Chin. J. Org. Chem. 2012, 32, 632 (in Chinese).

(邬旭然, 刘超, 杨泽, 姚楠楠, 王进军, 有机化学, 2012, 32, 632.)

[14] Ji, J.-Y.; Xia, S.-W.; Zhao, L.-L.; Li, J.-Z.; Qi, C.-X.; Wang, J.-J. Chin. J. Org. Chem. 2013, 33, 1457 (in Chinese). (纪建业, 夏尚文, 赵丽丽, 李家柱, 祁彩霞, 王进军, 有机化学, 2013, 33, 1457.)

[15] Smith, K. M.; Gogg, D. A.; Simpson, D. J. J. Am. Chem. Soc. 1985, $107,4946$.

[16] Wang, J.-J.; Li, Y.-W.; Li, J.-Z.; Yin, J.-G. Chin. J. Org. Chem. 2011, 31, 2074 (in Chinese)

(王进军, 李韵伟, 李家柱, 殷军港, 有机化学, 2011, 31, 2074.) 